\title{
IR-spectra of Halogenidanil under High Pressure: Metallization of Iodanil by Charge Transfer
}

\author{
A. Nakayama, K. Aoki, and I. Shirotani* \\ National Institute of Materials and Chemical Research, Tsukuba, Ibaraki 305, Japan \\ ${ }^{*}$ Muroran Institute of Technology, 27-1, Mizumoto, Muroran-shi 050, Japan
}

\begin{abstract}
Infrared (IR) spectra of tetraiod-p-benzoquinone (iodanil) and tetrabromid-p-benzoquinone (bromanil) have been measured to $43 \mathrm{GPa}$ at room temperature. Reversible spectral change below $43 \mathrm{GPa}$ and softening behavior of $\mathrm{C}$-I and $\mathrm{C}=\mathrm{O}$ stretching modes observed for iodanil indicate that the enlargement of charge transfer interactions of $\mathrm{O} \cdots \mathrm{I}$ and $\mathrm{I} \cdots \mathrm{I}$ between the adjacent molecules. Bromanil shows no softening behavior. Significant peak broadening takes place above $24 \mathrm{GPa}$, indicating polymerization.

[iodanil, pressure-induced metallization, superconductivity, infrared spectroscopy, charge transfer interaction]
\end{abstract}

\section{Introduction}

Tetraiod-p-benzoquinone (iodanil) is a rare monomer crystal showing metallization at room temperature and $30 \mathrm{GPa}^{1}$, and superconductivity at $2 \mathrm{~K}$ and $52 \mathrm{GPa}^{2}$ The metallization pressure is the lowest in a series of polycyclic aromatic crystals. ${ }^{3}$ In addition, the superconductive behavior is expected as the first observation for monomer crystals including their high-pressure phases. However, the detail of metallization has not been clarified. One essential question is molecularity in the metallic and superconductive phases. No experimental evidence for the metallic molecular solid has been reported and there still remains an possibility that the metallization and superconductivity arise from metallic solid phase of iodine ${ }^{4}$ deposited from decomposed iodanil under high pressure.

In this study, the pressure dependences of infrared (IR) spectra of iodanil and tetrabromid-p-benzoquinone (bromanil) were measured at room temperature. IR spectroscopy is a very effective tool to investigate high pressure bonding states, since the vibrational frequencies are directly affected by their changes with pressure. The study of bromanil obtained by replacing all four iodine atoms of iodanil with bromine atoms is useful for systematic understanding of the bonding states and bulk properties of iodanil.

\section{Experimental}

Sample preparation was carried out as follows. 5 Bromanil was purified by sublimation with pumping and recrystallization. Iodanil was synthesized by refluxing bromanil first with potassium iodine, and then, with sodium iodine in hot alcohol. The product was purified by sublimation with pumping and recrystallization. Purity of the sample was checked by mass spectra.

IR spectra were measured with a small diamond anvil cell (DAC) with $0.3 \mathrm{~mm}$-culet-diameter and $1 \mathrm{~mm}$-thick diamond anvils. The gasket was made of $40 \mu \mathrm{m}$-thick stainless steel, in which a $125 \mu \mathrm{m}$-hole was drilled. No pressure transmitting fluid was used to make a same condition as that for the measurement of the electrical resistance. ${ }^{2}$ The single crystals of iodanil and bromanil were used for IR measurement as for electrical resistance measurements. IR spectra were monitored at a pressure interval of 1 to $2 \mathrm{GPa}$ up to $43 \mathrm{GPa}$ beyond the metallic pressure of $32 \mathrm{GPa}$. IR spectra were also measured on unloading process.

Transmission IR spectra were measured by using a microscope FT-IR spectrometer. Substantial spectra of the samples were obtained by subtracting the reference spectrum of the empty DAC measured at $1 \mathrm{~atm}$ from the original spectra. Pressure was determined by the ruby $R_{1}$ pressure scale $(0.365$ $\mathrm{nm} / \mathrm{GPa}$ ).

\section{Results}

The mass spectra of iodanil showed that $\mathrm{C}_{6} \mathrm{O}_{2} \mathrm{I}_{3}{ }^{79} \mathrm{Br}$ and $\mathrm{C}_{6} \mathrm{O}_{2} \mathrm{I}_{3}{ }^{81} \mathrm{Br}$ were slightly contained as impurities in the sample. We, however, used this sample, since the IR spectra of the intramolecular vibrations seemed not to be significantly disturbed from those of the impurities. The spectra of bromanil indicated no inclusion of impurities.

The pressure dependences of the peak frequencies of iodanil and bromanil are shown in Fig. 1. The peak frequencies are obtained by fitting lineshapes with a Lorentian function or roughly estimated from eye measured center positions for saturated peaks. The vertical axes indicate the peak frequency shift, $\omega_{0 b s}-\omega_{0}$, where $\omega_{\text {obs }}$ and $\omega_{0}$ are frequencies at measured pressure and ambient pressure, respectively. The values of $\omega_{0}$ for each absorption peak are given in Fig. 1 .

The IR absorption peaks of iodanil disappeared above 18 $\mathrm{GPa}$, while reflection intensity began to increase. ${ }^{6}$ The absorption peaks appeared again when the pressure was reduced to $18 \mathrm{GPa}$ from $43 \mathrm{GPa}$ of the highest pressure reached. All of the peak frequency shifts showed reversible and proportional behaviors to pressure. The peaks with the frequencies of 1524 , $1176,1021,880$, and $582 \mathrm{~cm}^{-1}$ at ambient pressure showed positive frequency shifts with pressures. The frequency shift of the $\mathrm{C}=0$ stretching mode with $1660 \mathrm{~cm}^{-1}$ for $\omega_{0}$ showed slight negative pressure dependence. The $\mathrm{C}$-I stretching mode with $\omega_{0}$ 
$=695 \mathrm{~cm}^{-1}$ showed a large negative frequency shift. The observed vibrational peaks were classified into two categories based on their pressure dependence of the peak shifts: (1) antisymmetric $\mathrm{C}=\mathrm{C}$ and $\mathrm{C}-\mathrm{C}$ stretching modes, and $\mathrm{C}-\mathrm{C}-\mathrm{C}$ outof-plane bending mode showing positive frequency shifts, (2) antisymmetric $\mathrm{C}=\mathrm{O}$ and $\mathrm{C}$-I stretching modes showing negative frequency shifts.

(a) lodanil

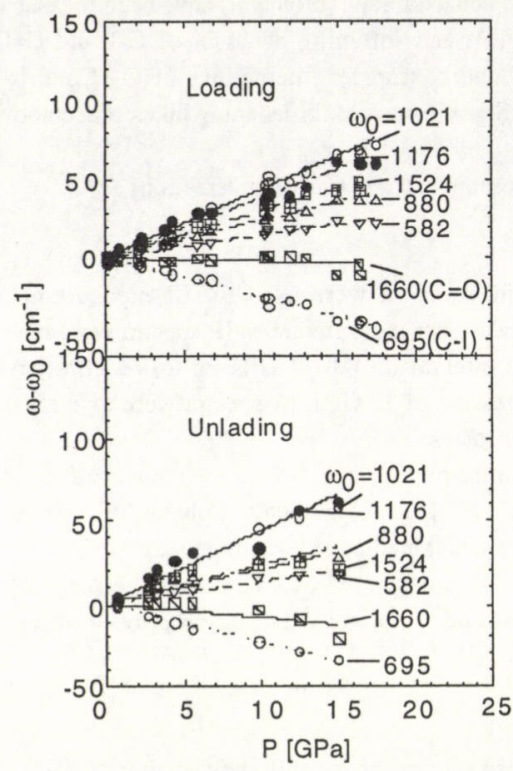

(b) Bromanil

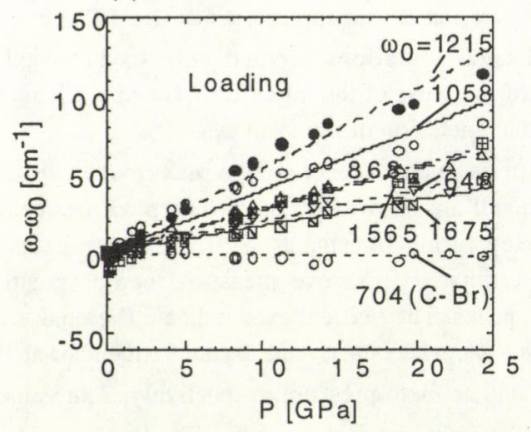

Fig. 1. Peak frequency shifts of iodanil in loading and unloading process (a) and bromanil in loading process (b).

The spectra of bromanil were deformed drastically above 19 $\mathrm{GPa}$. A remarkable upheaval of baseline was observed at 24 $\mathrm{GPa}$. The structure of IR spectrum was completely depressed above $36 \mathrm{GPa}$. Above $41 \mathrm{GPa}$ the spectra showed little change. All of the peak frequency shifts showed irreversible pressure dependences. The peak frequency shifts except that of the $\mathrm{C}-\mathrm{Br}$ stretching mode with $\omega_{0}=704 \mathrm{~cm}^{-1}$ were proportional to pressure with positive slopes. The $\mathrm{C}-\mathrm{Br}$ stretching frequency was insensitive to pressure.

\section{Discussion}

The metallic behavior of iodanil at the high pressure is discussed from IR spectra. The IR spectra give evidences of the metallization and the molecularity at high pressure: (1) no transmitted infrared light through the sample above $18 \mathrm{GPa}$, (2) the reversible spectral change with pressure. The spectral change with pressure below $18 \mathrm{GPa}$ corresponds to the variation of electrical resistance with pressure. Temperature dependence of the resistance for pressures from 17 to $27 \mathrm{GPa}$ indicates semiconductive behavior. Magnitude of band gap estimated at each pressure decreases with increasing pressure. The band gap remains at about $100 \mathrm{meV}$ at $18 \mathrm{GPa} .{ }^{7}$ No transmitted light in IR region at the corresponding pressure, therefore, may be attributed not to the band gap closure but to reduction of skin depth arising from increasing conduction electrons. Reversibility in IR spectrum and resistance measurements in loading and unloading process suggests that iodanil molecules persist without dissociation in its metallic phase above $18 \mathrm{GPa}$. It is supposed that the molecular dissociation is avoided by softening of the $\mathrm{C}=\mathrm{O}$ and $\mathrm{C}$-I bonds terminating the quinone ring.

The pressure dependences of the peak frequency shifts are analyzed. A vibrational frequency, $\omega_{\mathrm{obs}}$, at a pressure of $\mathrm{P}$ is able to be described by an initial frequency at ambient pressure, $\omega_{0}$.

$\omega_{\mathrm{obs}}=\omega_{0}+\frac{\Delta \omega}{\Delta \mathrm{P}} \times \mathrm{P}$

The pressure coefficient, $\frac{\Delta \omega}{\Delta \mathrm{P}}$, determined from the slopes of the frequency shifts with pressure is given in Fig. 1. This coefficient reflects change in the bonding state by loading pressure.

The $\mathrm{C}=\mathrm{C}$ and $\mathrm{C}-\mathrm{C}$ stretching modes and the $\mathrm{C}-\mathrm{C}-\mathrm{C}$ out-ofplane bending mode show positive values of $\frac{\Delta \omega}{\Delta P}$ indicating hardening of the carbon-born structure in the quinone ring. The hardening of the quinone ring produces increase of electroncloud overlapping, resulting in the conductive domain with high electron density. The negative values of $\frac{\Delta \omega}{\Delta P}$ for the $\mathrm{C}=\mathrm{O}$ and C-I stretching modes mean the softening of their bonds. Such softening is caused by acceleration of charge transfer interaction $^{8,9}$ of $\mathrm{O} \cdots \mathrm{I}$ and $\mathrm{I} \cdots \mathrm{I}$ between the adjacent molecules. The electron state modified the charge transfer interaction is explained by a dative structure $>\mathrm{C}=\mathrm{O}^{\delta+} \ldots \mathrm{I}^{\delta-}-\mathrm{C}<$. The charge transfer interaction makes the intermolecular conductive pass which is extended by loading pressure.

The extension of the conductive network is considered from the crystal structure of iodanil. Projection of the structure along 
the b-axis of iodanil at room temperature and atmospheric pressure is given in Fig. 2. The inter-atomic distances smaller than sum of van der Waals radii, are represented by dashed (A) and (B) bars with equivalent lengths. Molecule (2) is placed in the center of bc-plane and surrounded by molecules (1) and (3) located in the four corners of the bc-plane. Dashed bars (c) represent two different interatomic distances, 3.817 and 4.161 $\AA$. The former is smaller than the sum of van der Waals radii, and the later is $6 \%$ larger than that. These unequivalent $\mathrm{I} \cdots \mathrm{I}$ distances are expected to become equivalent when iodanil molecules approach enough at high pressure. It is supposed that this structural change leads to formation of conductive network as observed in iodine crystal. Compression of iodine causes the nearest-neighbor interatomic distance to approach the intramolecular one at $21 \mathrm{GPa} .{ }^{10}$ This structural change results in the metallization by the formation of two-dimensional network. In the case of iodanil, the changes in IR spectra and electric resistance with pressure imply the approach to the equivalent I . . I distance. Such molecular approach seems to be responsible for formation of three dimensional conductive network and hence the metallization of idoanil.

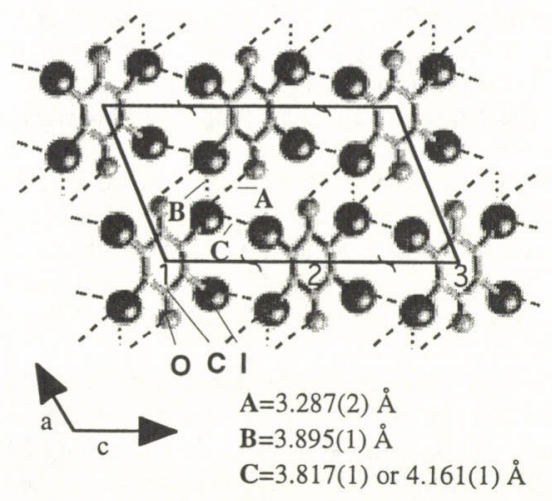

Fig. 2. Projection of the structure along the b-axis of iodanil.

Irreversible IR spectral change and line broadening observed for bromanil suggest molecular decomposition or polymerization. The peak frequency shift gives positive $\frac{\Delta \omega}{\Delta \mathrm{P}}$ for the modes related to the vibration of the carbon born structure of the quinone ring and the $\mathrm{C}=\mathrm{O}$ stretching mode except the $\mathrm{C}-\mathrm{Br}$ stretching mode. The value of the $\mathrm{C}-\mathrm{Br}$ stretching mode is almost zero. These values for carbon-born structure are similar pressure behaviors, which are comparable to those of iodanil. It is supposed to be related to increase of the electron density on the quinone ring. On the other hand, the $\mathrm{C}=\mathrm{O}$ and $\mathrm{C}-\mathrm{Br}$ stretching modes are different from those for the $\mathrm{C}=\mathrm{O}$ and $\mathrm{C}$-I stretching modes of iodanil, showing little shifts of the frequencies. The pressure insensitive behaviors of the
$\mathrm{C}=\mathrm{O}$ and $\mathrm{C}-\mathrm{Br}$ stretching modes suggest that bromanil has no dative structure 8,9 as shown in iodanil. According to the crystal structure of bromanil, ${ }^{11}$ all of the atomic distances between the neighbor molecules are larger than the sum of the van der Waals radii, suggesting no charge transfer interaction.

The increase of the conduction inside domain and the enlargement of the conductive network among them are speculated as a model for the metallization mechanism from the observed IR spectra, which indicate no transmission of infrared light through the sample above $18 \mathrm{GPa}$ and remarkable softening of $\mathrm{C}=\mathrm{O}$ and $\mathrm{C}-\mathrm{I}$ bonds arising from the charge transfer interactions of $\mathrm{O} \cdots \mathrm{I}$ and $\mathrm{I} \cdots \mathrm{I}$. The metallization and the absence of molecular dissociation of iodanil suggested by the reversible spectral change is a novel phenomenon for organic molecular crystals.

\section{Acknowledgement}

The authors are grateful to Dr. Masuhiro Mikami and Dr. Yoshitaka Matsushita of National Institute of Materials and Chemical Research, Mr. Masao Saito and Professor Kiichi Amaya of University of Osaka, for their collaboration and numerous discussions. Invaluable help for the experiment from Dr. Hiroshi Yamawaki, Dr. Takako Nakamura, Mr. Tsuguyori Ohana, and Mr. Masaya Moriyama of National Institute of materials and Chemical Research are gratefully acknowledged.

\section{Reference}

[1] I. Shirotani, A. Onodera, Y. Kamura, H. Inokuchi, and N. Kawai, J. Solid State Chem., 18, 235 (1976).

[2] T. Yokota, N. Takeshita, K. Shimizu, K. Amaya, A. Onodera, I. Shirotani, and S. Endo, Czechoslovak J. Phys., 46, 817 (1996).

[3] B. M. Riggleman, and H. G. Drickamer, L. Chem. Phys., 38, 2721 (1963).

[4] K. Shimizu, N. Tamitani, N. Takeshita, M. Ishizuka, K. Amaya, and S. Endo, Physica B, J. Phys. Soc. Jpn., 16, 1185 (1961).

[5] H. A. Torrey, and W. H. Hunter, J. Amer. Chem. Soc., 34, 702 (1912).

[6] A. Nakayama, K. Aoki, and I. Shirotani, priate communication.

[7] A. Nakayama, K. Aoki, M. Saito, and K. Amaya, priate communication.

[8] D. L. Glusker, H. W. Tompson, and R. S. Mulliken, J. Chem. Phys., 21, 1407 (1953).

[9] H. Yamada, and K. Kojima, J. Amer, Chem. Soc., 82, 1543 (1960).

[10] K. Takemura, S. Minomura, O. Shimomura, Y. Fujii, and J. D. Axe, Phys. Rev. B, 26, 998, (1982).

[11] I. Ueda, J. Phys. Soc. Jpn., 16 1185, (1961). 